\title{
Differences in artificial ligament graft osseointegration of the anterior cruciate ligament in a sheep model: a comparison between interference screw and cortical suspensory fixation
}

\author{
Hong Li", Fangyi Jiang ", Yunsheng Ge", Fang Wan, Hongyun Li, Shiyi Chen \\ Department of Sports Medicine, Huashan Hospital, Shanghai, China \\ Contributions: (I) Conception and design: H Li, F Jiang, Y Ge, S Chen; (II) Administrative support: S Chen; (III) Provision of study materials or \\ patients: H Li, H Li, F Wan; (IV) Collection and assembly of data: H Li, F Jiang, H Li, Y Ge; (V) Data analysis and interpretation: H Li, F Jiang, Y \\ Ge; (VI) Manuscript writing: All authors; (VII) Final approval of manuscript: All authors. \\ \#These authors contributed equally to this work. \\ Correspondence to: Shiyi Chen, MD, PhD. No. 12, Wulumuqi Zhong Road, Shanghai 200040, China. Email: cshiyi@163.com.
}

Background: Interference screws are the most common femoral fixation for anterior cruciate ligament (ACL) reconstruction with polyethylene terephthalate (PET) artificial ligaments. However, interference screws have several disadvantages, such as the risk of one tunnel blowout and damage to the graft. Suspensory fixations have the advantages of high tensile strength and promotion of graft bone contact. The purpose of this study was to compare PET artificial ligament graft osseointegration between interference screw fixation (ISF) and cortical suspensory fixation (CSF) for ACL reconstruction.

Methods: Forty sheep underwent ACL reconstruction of the right knee with PET artificial ligament. The graft was fixed with ISF or CSF for femoral fixation. Animals were randomly assigned to the ISF ( $\mathrm{n}=20$ ) or the CSF ( $\mathrm{n}=20$ ) groups. The sheep were sacrificed at 3 or 12 months postoperatively for biomechanical tests, micro computed tomography (micro-CT) scans, and histological assessments.

Results: The mean load-to-failure between the CSF group $(836 \pm 355 \mathrm{~N})$ appeared higher than that of the ISF group $(604 \pm 277 \mathrm{~N})$ at 3 months, but no significant difference was detected between the groups $(\mathrm{P}=0.24)$. At 12 months, there was also no significant difference in load-to-failure between the CSF and ISF groups $(1,194 \pm 350$ vs. $1,097 \pm 764 \mathrm{~N} ; \mathrm{P}=0.78)$. According to the micro-CT scan results, the femoral bone tunnel diameter of the ISF group appeared larger than that of the CSF group at 3 months $(12 \pm 1 v s .10 \pm 1 \mathrm{~mm}$; $\mathrm{P}=0.02)$ and similar to that of the CSF group at 12 months $(12 \pm 1$ vs. $11 \pm 2 \mathrm{~mm} ; \mathrm{P}=0.38)$. Furthermore, histological results showed that at the graft-tunnel interface of the femoral tunnel aperture, disoriented fibers formed in the ISF group while oriented and dense fibers formed in the CSF group.

Conclusions: ACLR with synthetic ligament by cortical suspension devices with adjustable loops demonstrated a better graft-bone healing capacity at the femoral tunnel aperture compared with that from titanium interference screws over 12 months postoperatively. No significant difference was found in biomechanical strength between the two fixation methods during the early healing stage.

Keywords: Anterior cruciate ligament (ACL); artificial ligament; cortical suspensory fixation (CSF); interference screw fixation (ISF); graft osseointegration

Submitted Mar 07, 2021. Accepted for publication Jul 14, 2021.

doi: 10.21037/atm-21-1076

View this article at: https://dx.doi.org/10.21037/atm-21-1076 


\section{Introduction}

Synthetic ligaments have emerged as substitute materials for allografts or autografts in surgical anterior cruciate ligament reconstruction (ACLR). The benefits of synthetic ligaments are the elimination of donor-site morbidity and postoperative early rehabilitation (1-4). Most clinical studies have demonstrated satisfying long-term outcomes after ACLR using synthetic grafts (5-8). However, a few followup studies for synthetic graft reconstruction showed some failure cases, including the graft being pulled or loosened away from the bone (9-12). The incidence of synthetic ligament failure, which could result from inferior graft osseointegration, was also slightly higher in large animal studies (13). Accordingly, understanding how to guarantee firm fixation of the reconstructed graft and subsequent graft osseointegration is important for using synthetic ligaments in ACLR (14).

As reported $(15,16)$, improper graft fixation can lead to knee laxity or constraint and graft slippage, as well as delayed graft osseointegration and tunnel widening. These can lead to graft stress failure (17). The importance of proper graft fixation is greater when reconstructed using synthetic grafts, as early mobilization is usually expected. Interference screw fixation (ISF) is commonly applied in synthetic ligament reconstruction (18-20). However, it is still doubtful whether ISF is the optimal choice. ISF presents several disadvantages, including the risk of bone tunnel blowout as well as damage to the grafts. Furthermore, screw divergence during surgical reconstruction may lead to inadequate graft fixation strength during the early range of motion exercise (21-23). Conversely, when applied as the femoral fixation method, cortical suspensory fixation (CSF) shows the advantages of the precise tunnel positioning, high tensile strength, and avoidance of graft damage (24). In autograft ACLR, CSF has proven superior for graft healing compared with ISF (25-27). To date, a comparison between CSF and ISF in synthetic ligament ACLR has not been reported.

Therefore, the purpose of this study was to compare PET artificial ligament graft osseointegration between ISF and CSF for ACL reconstruction in a sheep model. Sheep have been widely used to assess postoperative results in ACLR and have shown their validity in evaluating biomechanical and histological properties (28-30). We hypothesized that biomechanical strength and graft osseointegration after synthetic ligament ACLR by CSF would be superior to those by ISF. We present the following article in accordance with the ARRIVE reporting checklist (available at https:// dx.doi.org/10.21037/atm-21-1076).

\section{Methods}

\section{Study design}

The animal experiments were performed under a project license (No. 201401050001) granted by the Institutional Animal Care and Use Committee (IACUC) of Shanghai Jiao Tong University Animal Department, in compliance with national guidelines for the care and use of animals. A total of 40 healthy adult sheep ( $n=4$ males; $n=16$ females) were used. The sheep were randomly assigned to two groups ( $n=20$ per group), with an equal sex ratio. In the ISF group, grafts were fixed with titanium interference screws on the femoral side. In the group, CSF grafts were fixed with cortical suspension devices using an adjustable loop (ACL TightRope Implant, produced by Arthrex Inc., Florida) on the femoral side. Ten sheep were euthanized at 3 or 12 months after surgery in each group. Gross morphology was evaluated immediately after euthanasia and arthrotomy. Afterwards, micro-CT and biomechanical testing were conducted in six sheep from each group. Histological evaluation of the intraarticular and intraosseous portions of the graft on the femoral side was performed in four sheep from each group.

\section{Surgical procedure}

A unilateral open surgical procedure was performed under general anesthesia (anesthesia was induced with $4 \mathrm{mg} / \mathrm{kg}$ propofol. Anesthesia was maintained with $2 \%$ isoflurane). A medial arthrotomy was performed, and the patella was laterally displaced. The fat pad was partly excised to expose the anterior cruciate ligament (ACL). The knee joint was then flexed to $90^{\circ}$. The ACL was clearly exposed and cut at the midpoint of the intra-articular part of the ligament with the remnant preserved. Femoral and tibial tunnels were drilled with guide through the center of the ACL stump. The bone tunnel diameter was $7.0 \mathrm{~mm}$.

In the ISF group, a synthetic ligament [polyethylene terephthalate (PET) ligament (Ligatech Bioscience Co. Ltd., Shanghai), diameter $=7 \mathrm{~mm}$, single-stranded] was inserted through the tunnel and fixed on the femoral side with a titanium interference screw (diameter: $7 \mathrm{~mm}$; length: $25 \mathrm{~mm}$ ) using the outside-in method. In the CSF group, a $7.0 \mathrm{~mm}$ diameter femoral bone socket with a tunnel length of $15 \mathrm{~mm}$ was drilled inside-out. The synthetic graft [PET ligament 

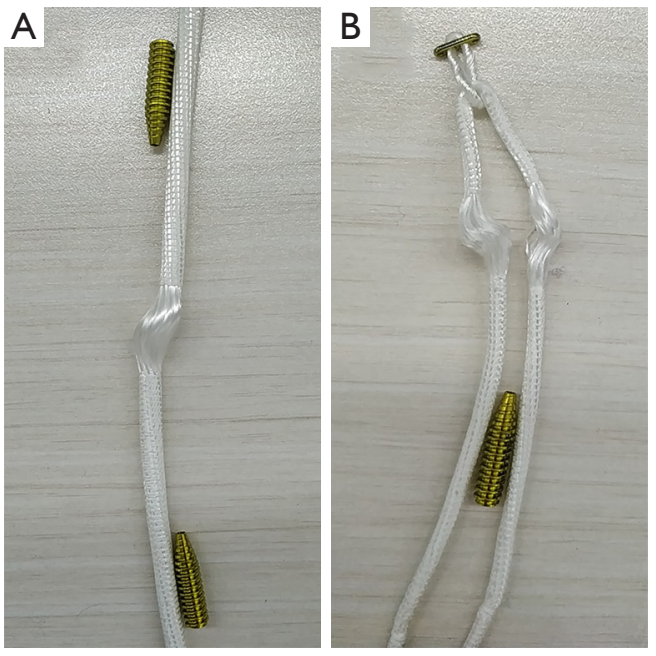

Figure 1 Graft is fixed with interference screw fixation (A) or cortical suspensory fixation (B) for the femoral fixation.

(Ligatech Bioscience Co. Ltd., Shanghai), diameter = $7 \mathrm{~mm}$, double-stranded] was fixed with ACL TightRope Implant (Figure 1). The grafts were tensioned by 30 knee flexion-extension cycles, and no obvious graft-tunnel motion was observed. On the tibial side, the graft was fixed with an interference screw (diameter: $7 \mathrm{~mm}$; length: $25 \mathrm{~mm}$ ). After fixation, the redundant extremities of the ligament were cut flush with the bone surface. After surgery, the animals were kept in cages the entire time and walked freely before sacrifice, with no restriction on active weight-bearing. All sheep were euthanized at 3 or 12 months postoperatively. Anesthesia was induced with Zoletil $(25 \mathrm{mg} / \mathrm{kg}$, Virbac, France) and maintained with propofol $(10 \mathrm{~mL} / \mathrm{kg})$, followed by intravenous injection with $10 \%$ potassium chloride $(1 \mathrm{mg} / \mathrm{kg})$. After euthanasia, the articular capsule was opened, and the joint cavity was exposed for gross observation. Operated knees were dissected with the femoral side preserved at least $10 \mathrm{~cm}$ above the adductor tubercle and the tibial side preserved at least $10 \mathrm{~cm}$ beneath the tibial tuberosity for further testing. Biomechanical testing and CT scans were conducted on the day of dissection on unfixed specimens (6 samples for each group at each time point). The rest of the specimens (4 samples for each group at each time point) were immediately fixed in $10 \%$ neutral buffered formalin liquid for histological evaluation.

\section{Micro-computed tomography (CT) analysis}

After sacrifice, the graft-femoral samples $(n=6)$ were scanned with micro-CT (Brilliance CT, Model No. 728302; Philips Medical Systems, Inc., Cleveland, Ohio) before the biomechanical studies. All bone tunnel model images were acquired via a Philips Brilliance workstation (Software Version: 3.2.4.1900; Philips Medical Systems, Inc., Cleveland, Ohio). The bone tunnel diameter at the aperture was analyzed with DICOM software according to a previous method (Figure 2) (31). In each image, the tunnel diameter was measured from two directions and averaged. The femoral tunnel diameter of each case was calculated as the average of the values of five consecutive images.

\section{Biomechanical testing}

The ends of femoral and tibial specimens $(n=6)$ were infiltrated and embedded in a cup with PMMA for 10 minutes, during which a tension-free intraarticular ligament was ensured. The fixation devices were not removed prior to testing. All soft tissues were carefully removed from the tunnel-graft complex. Mechanical testing was conducted using a materials testing machine (Fatig-A-ECO101). The embedded tibial and femoral ends were firmly fixed with clamps. The knee joint was placed in extension and tested parallel to the longitudinal femoral axis. A preload of $1 \mathrm{~N}$, followed by loading with a displacement rate of $10 \mathrm{~mm} /$ minute, was applied to the sample until the graft was pulled out from the bone tunnel or the intraarticular portion ruptured. The ultimate tensile strength, load to failure, ultimate displacement, and stiffness were obtained from the force-displacement curve.

\section{Histological analysis}

The graft-femoral samples were fixed in $10 \%$ neutral buffered formalin liquid for histological evaluation. The uncalcified femoral bone block containing a complete bone tunnel was embedded in PMMA. The bone block was sectioned into $50 \mu \mathrm{m}$ thick slices parallel to the longitudinal axis of the bone tunnel. The intraosseous portion was stained with Paragon dye. The intraarticular portion was transversely sectioned into $5 \mu \mathrm{m}$-thick slices and stained with hematoxylin and eosin (H\&E).

To assess the intraosseous portion of the graft, a panorama of the femoral bone tunnel was created using Adobe Photoshop CC 2018 (Adobe Systems, San Jose, CA) to observe the histological morphology of the graft and graft-bone interface. Graft osseointegration was evaluated further at high magnification. The bone tunnel was divided 


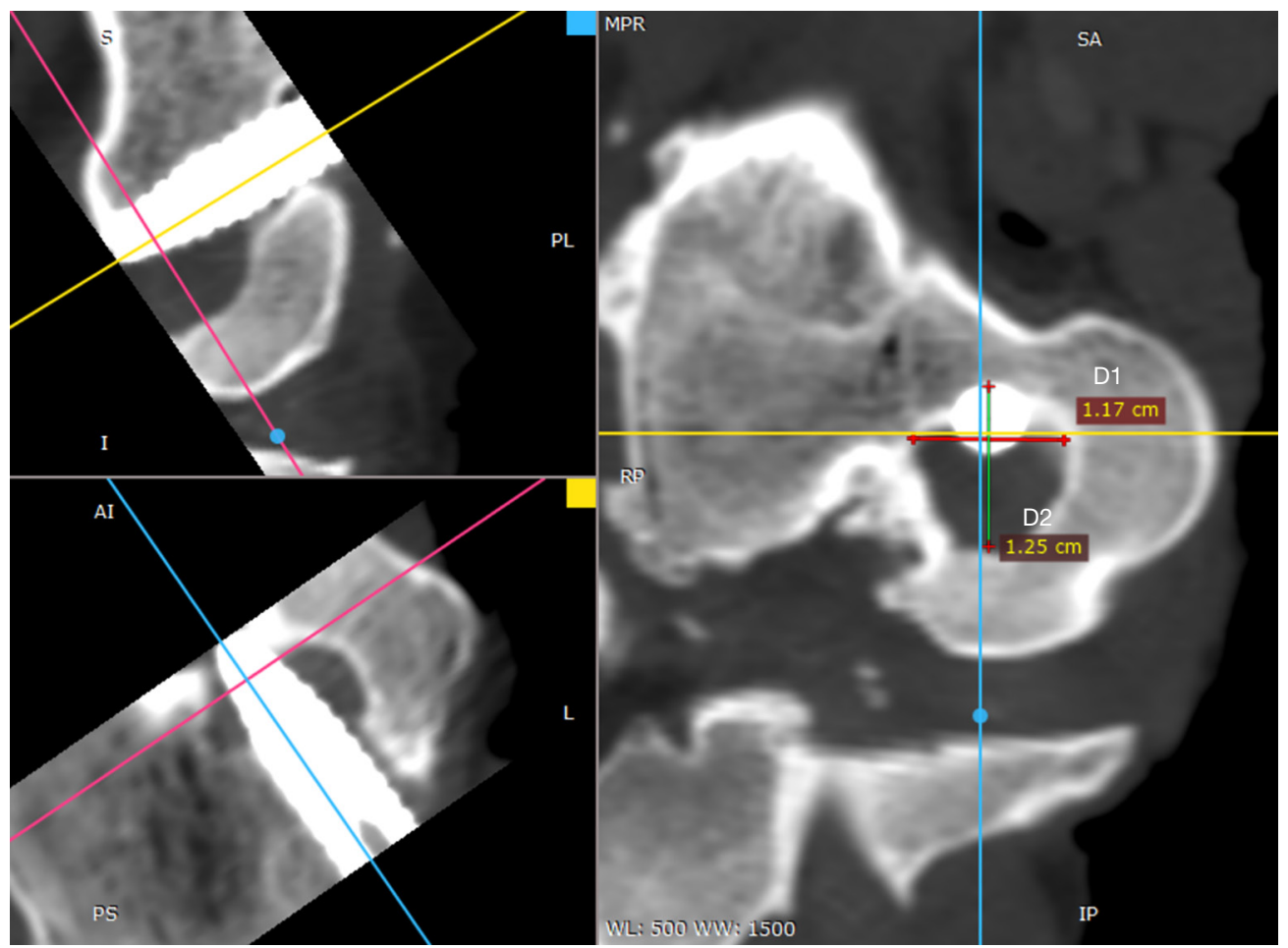

Figure 2 Measurement of the femoral bone tunnel diameter at the aperture. On the right image, the bone tunnel circular plane is perpendicular to the long axis of the femoral tunnel. The bone tunnel diameter was calculated using the following equation: (D1 + D2)/2.

into the tunnel aperture and tunnel exit by the midpoint of the tunnel. Tunnel aperture and exit were evaluated separately. The intraarticular sections were evaluated for fibrous ingrowth, neovascularization, and cell infiltration into grafts.

\section{Statistical analysis}

Statistical analysis was performed with SPSS Statistics 25 (SPSS Inc., Chicago, IL). Continuous data were presented as mean \pm standard deviation. Nonparametric MannWhitney test was used for comparison of ultimate loadto-failure between two groups. Unpaired $t$-test was used for comparison of load to failure and stiffness between two groups. The level of statistical significance was set at 0.05 .

\section{Results}

\section{Gross observation}

After each time point, no graft failure or gross infection occurred. Grafts were intact in all specimens and available for further analysis. For synovial covering, there was no difference in the extent of synovial coverage between the CSF group and the ISF group (Figure 3).

\section{$C T \operatorname{scan}(n=6)$}

According to the micro-CT scan, the ISF group revealed a larger femoral bone tunnel than the CSF group at 12 months after surgery (Figure 4). The femoral bone tunnel diameter of the ISF group appeared larger than that of the CSF group at 3 months $(12 \pm 1$ vs. $10 \pm 1 \mathrm{~mm}$; $\mathrm{P}=0.02)$ and at 12 months $(12 \pm 1$ vs. $11 \pm 2 \mathrm{~mm} ; \mathrm{P}=0.38)$. In the tibial bone tunnel, there was no significant difference in bone tunnel diameter between the ISF and CSF groups at 3 months $(9 \pm 1$ vs. $8 \pm 2 \mathrm{~mm} ; \mathrm{P}=0.55)$ and 12 months $(9 \pm 1$ vs. $9 \pm 1 \mathrm{~mm} ; \mathrm{P}=0.86$ ) (Figure 5).

\section{Biomechanics $(n=6)$}

The mean load-to-failure between the CSF group $(836 \pm 355 \mathrm{~N})$ appeared higher than that of the ISF group $(604 \pm 277 \mathrm{~N})$ at 3 months, while no significant difference was detected between groups $(\mathrm{P}=0.24)$. At 12 months, there 

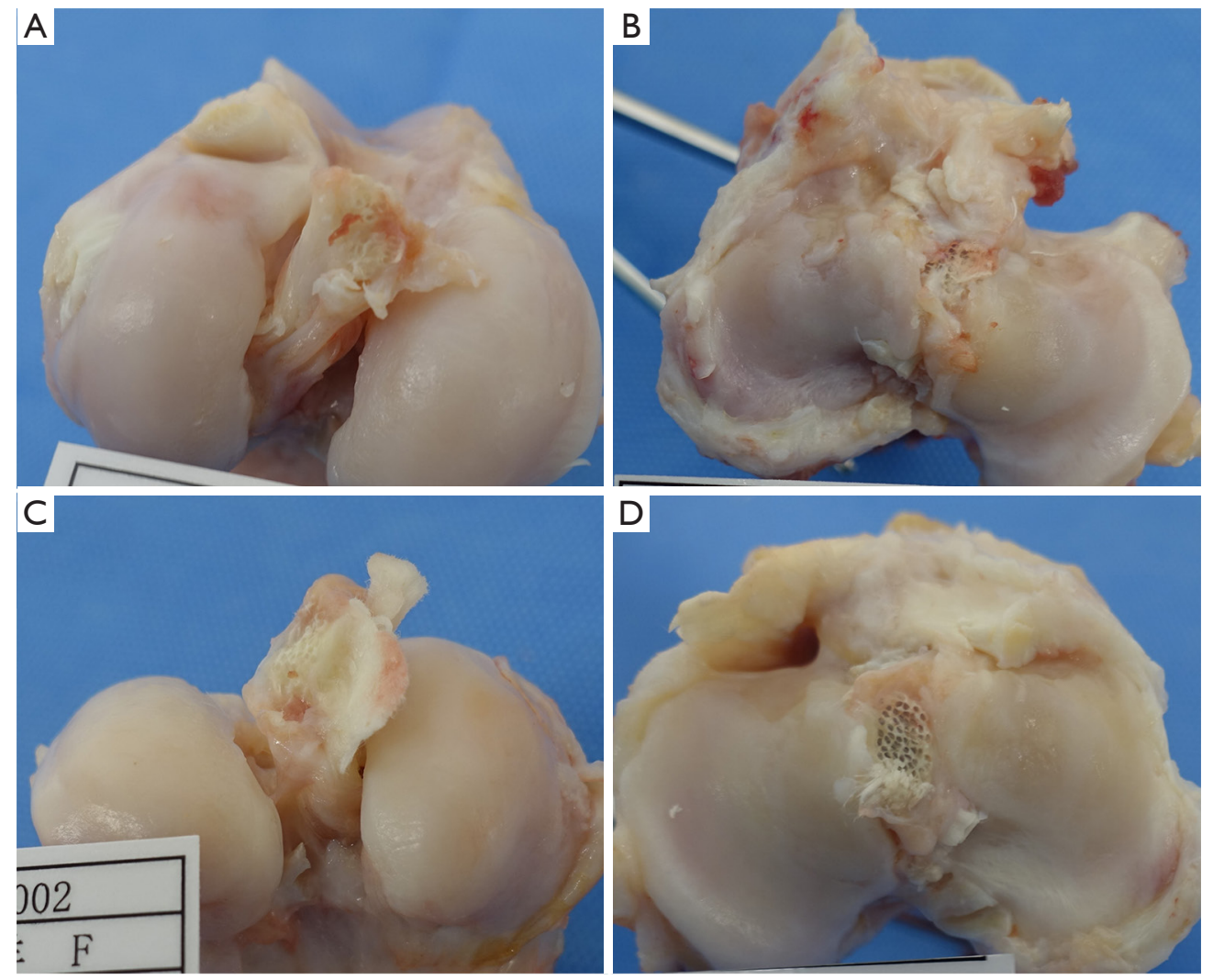

Figure 3 Gross morphological appearance of femoral-graft samples (A,C) and tibial-graft samples (B,D) between the CSF group (A,B) and the ISF group (C,D) at 12 months after surgery. ISF, interference screw fixation; CSF, cortical suspensory fixation.

was also no significant difference in load-to-failure between the CSF and ISF groups $(1,194 \pm 350 v s .1,097 \pm 764 \mathrm{~N}$; $\mathrm{P}=0.78)$. Furthermore, there was also no significant difference in the mean stiffness at 3 months $(147 \pm 38$ vs. $114 \pm 52 \mathrm{~N} / \mathrm{mm} ; \mathrm{P}=0.23)$ or at 12 months $(169 \pm 54$ vs. $205 \pm 97 \mathrm{~N} / \mathrm{mm} ; \mathrm{P}=0.44)$ between the CSF and ISF groups. Moreover, six contralateral healthy knee samples were utilized to test the biomechanical properties of the native ACL. The mean load-to-failure and stiffness of the native ACL were $1,254 \pm 350 \mathrm{~N}$ and $416 \pm 121 \mathrm{~N} / \mathrm{mm}$, respectively (Figure 6). At 3 months, all the grafts of the CSF group were removed from the tibial tunnel, and the grafts of the ISF group were removed from the femoral tunnel in two samples and from the tibial tunnel in four samples. At 12 months, the grafts of the CSF group were selected from the femoral tunnel in one sample, from the tibial tunnel in three samples, and from the graft rupture in two samples. The grafts of the ISF group were chosen from the femoral tunnel in one sample and from the tibial tunnel in five samples at 12 months (Figure 7).

\section{Histology $(n=4)$}

In the ISF group, while the tunnel exit was predominantly occupied by graft fibers, the tunnel aperture appeared to be infiltrated by more fibrous tissue at 3 months (Figure $8 \mathrm{~A}$ ). Large portions of synthetic material could still be observed at 3 months postoperatively in the femoral bone tunnel in the CSF group, as shown in Figure 8B. In addition, prominent tunnel enlargement was found in the tunnel aperture of group ISF (Figure $8 A$ ), whereas consistent tunnel widths from the tunnel aperture to exit were observed in group CSF (Figure 8B).

At 3 months after surgery, the histological appearance at high magnification also confirmed that the enlarged interface of the ISF group was primarily fibrous tissue. The collagen fibers were arranged irregularly, and prominent cellularization and vascularization were found in the area. Giant cells were observed around graft fascicles or at the graft-bone interface in 3/4 sections, indicating foreign body reactions in the enlarged area. A thin layer of newly formed 


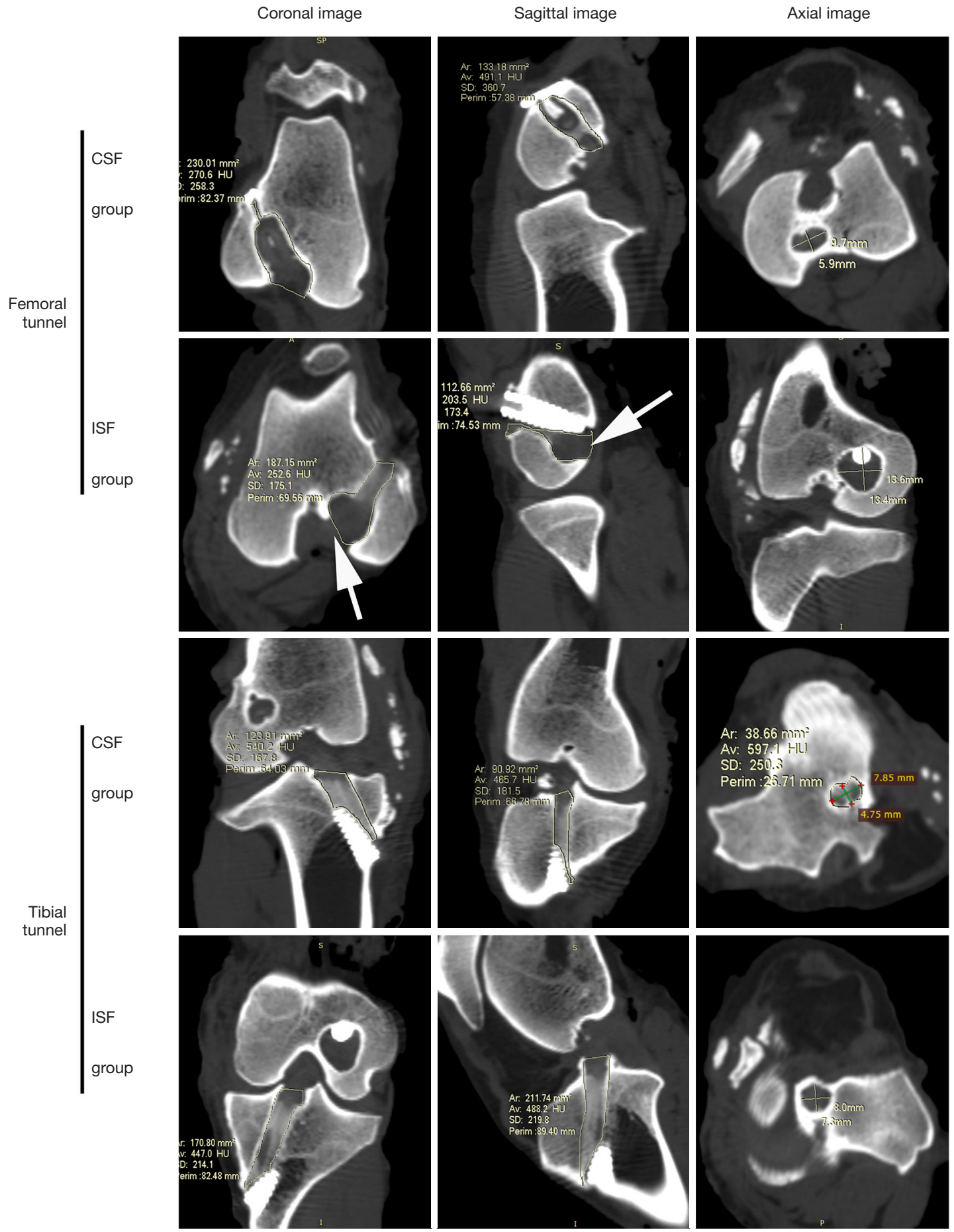

Figure 4 CT scan images between the CSF group and ISF group at 12 months after surgery. White arrows indicate femoral bone tunnel enlargement. ISF, interference screw fixation; CSF, cortical suspensory fixation; CT, computed tomography. 
A

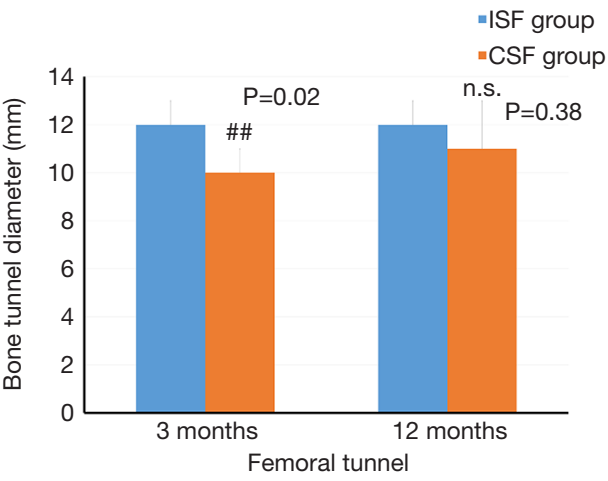

B

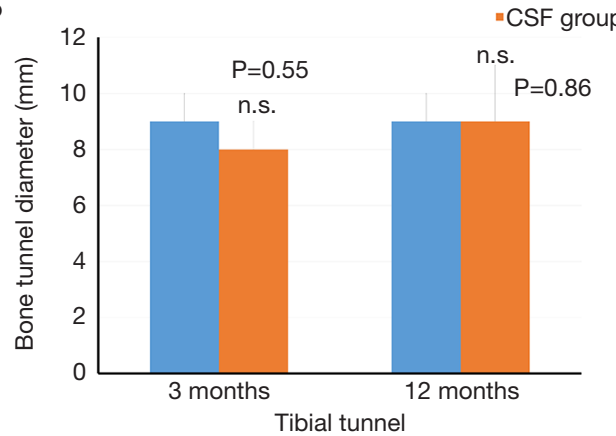

Figure 5 Comparison of femoral (A) or tibial (B) bone tunnel diameter between the CSF group and ISF group. ISF, interference screw fixation. CSF, cortical suspensory fixation. ${ }^{\# \text { indicates that }}$ there was a significant difference between the CSF group and ISF group. n.s. indicates that there was no significant difference between the CSF group and ISF group.

bone tissue could be observed in the tunnel aperture of all sections, indicating that the process of osteogenesis occurred in this region (Figure 9A). A fibrous interzone was also observed in the tunnel exit of the ISF group. However, the graft-bone interface was significantly narrower, and direct graft-to-bone contact in part of the interzone was found in 3/4 of the sections (Figure 9B). A fibrous capsule at the graft-bone interface was found in both the distal and tunnel exits in the CSF group. In two specimens, dense fibrous tissue was found in the tunnel aperture. The collagen fibers were regularly arranged and oriented perpendicular to the direction of the bone tunnel. The same region of the other sections in the CSF group retained disoriented collagen fibers. Of note, two sections in the CSF group exhibited the formation of fibrocartilage in part of the tunnel aperture (Figure 9C). The interface was also filled with disoriented collagen fibers in the vicinity of the tunnel exit (Figure 9D). Newly formed bone tissue was found adjoining the fibrous
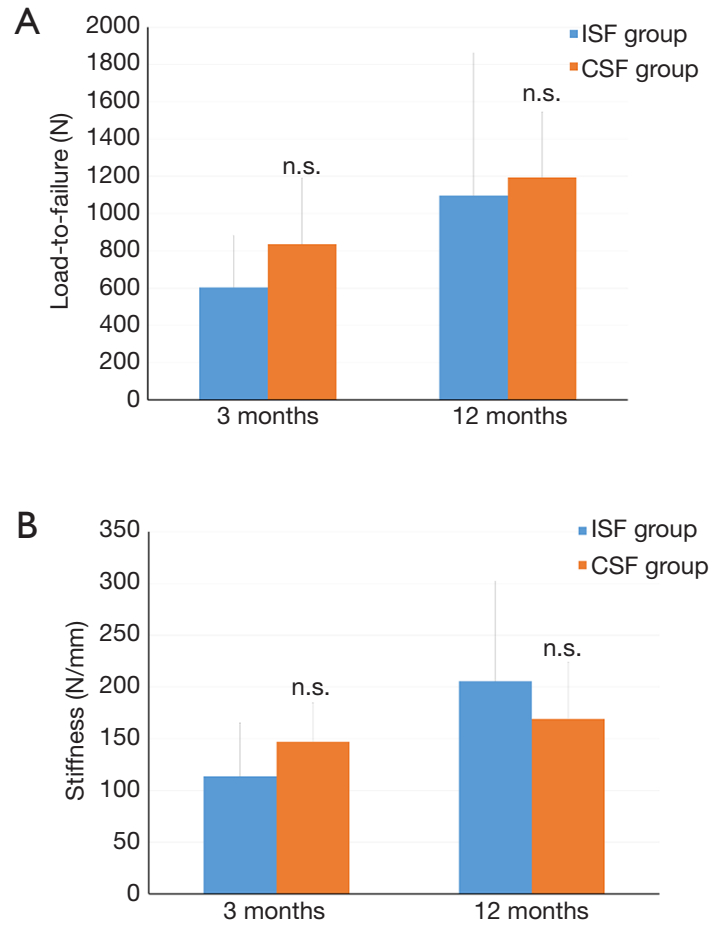

Figure 6 Comparison of biomechanical property between the CSF group and ISF group. (A) Ultimate load-to-failure; (B) stiffness. n.s. indicates that there was no significant difference between the CSF group and ISF group. ISF, interference screw fixation; CSF, cortical suspensory fixation.

tissue layer. Osteoclasts were rarely identified along the bone interface in either group (Figure 9C).

At 12 months after surgery, denser fibers formed at the proximal site of the femoral tunnel in the ISF group, and the fibers were parallel to the bone (Figure 10A). At the distal femoral tunnel, the graft was in direct contact with the bone surface, and new bone tissue protruding to the PET graft was observed in some specimens (Figure 10B). In the CSF group, the graft was in close contact with the host bone at the proximal and tunnel aperture of the femoral tunnel. Some oblique fibers, similar to Sharpey's fiber, were observed to link the graft and bone in the femoral tunnel of the CSF group (Figure 10C,10D).

\section{Discussion}

CSF has been gaining popularity for use in autograft ACLR. It has been proven to promote graft-bone healing process 

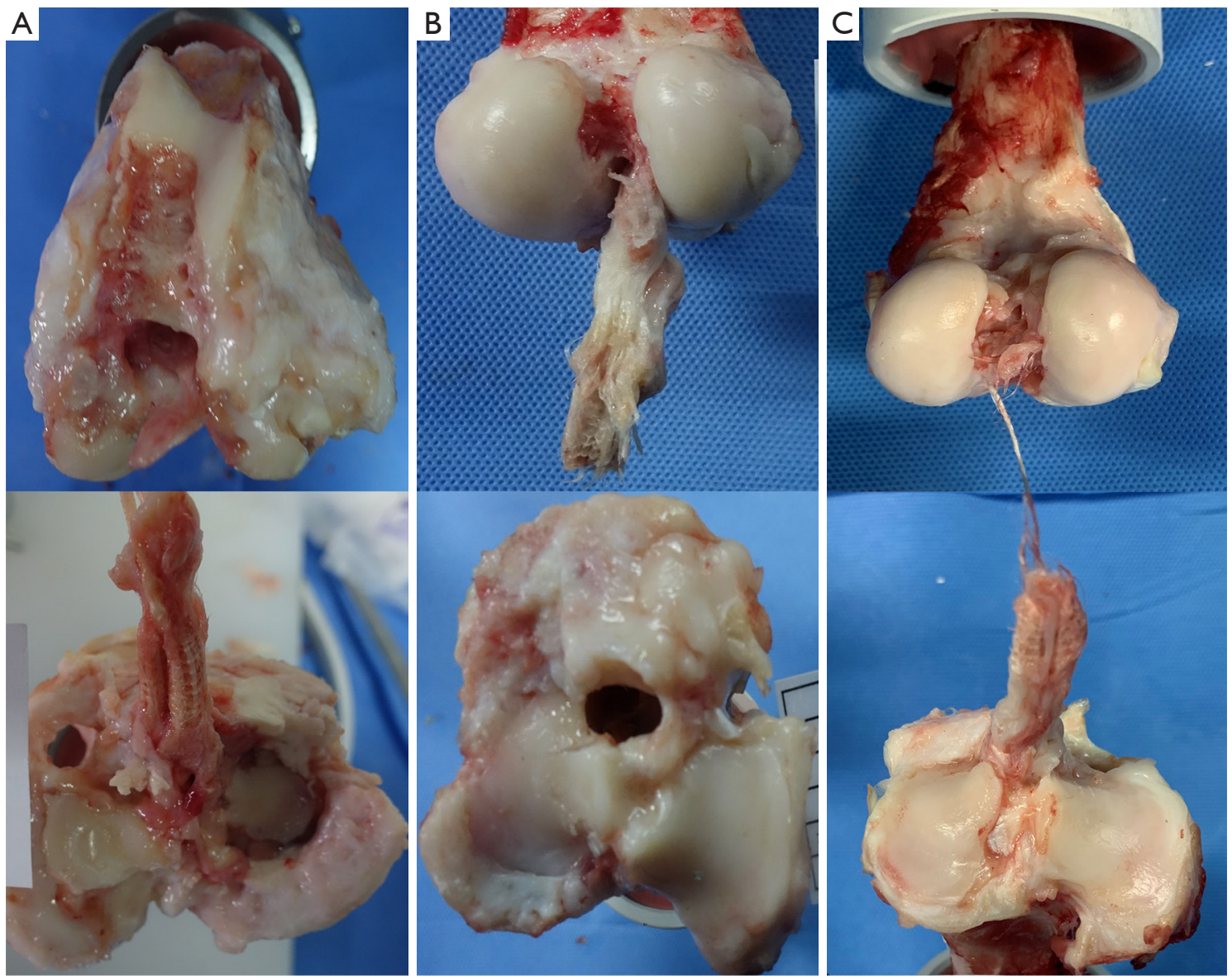

Figure 7 Graft failure models. (A) Graft was pulled out from the femoral tunnel; (B) graft was pulled out from the tibial tunnel; (C) graft was ruptured.
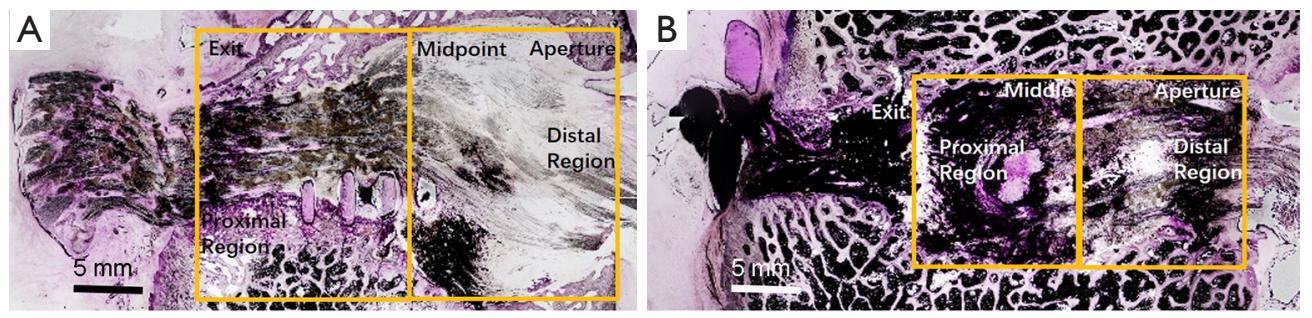

Figure 8 Photomicrographic panorama of graft-tunnel complex with Paragon staining at 3 months after surgery: (A) femoral tunnel of the ISF group, (B) femoral tunnel of the CSF group. The region between tunnel aperture and midpoint was named as tunnel aperture, and the region between tunnel midpoint and exit was named as tunnel exit. ISF, interference screw fixation; CSF, cortical suspensory fixation.

and reduce tunnel widening in comparison with ISF (26). Interference screws have several disadvantages, such as the risk of one tunnel blowout and damage to the graft. Suspensory fixations have the advantages of high tensile strength and promotion of graft bone contact. To date, a comparison between CSF and ISF in synthetic ligament ACLR has not been reported.

In this study, the mean load-to-failure was $836 \pm 355 \mathrm{~N}$ in the CSF group and $604 \pm 277 \mathrm{~N}$ in the ISF group at 3 months. At 12 months, the load-to-failure of the CSF and ISF 

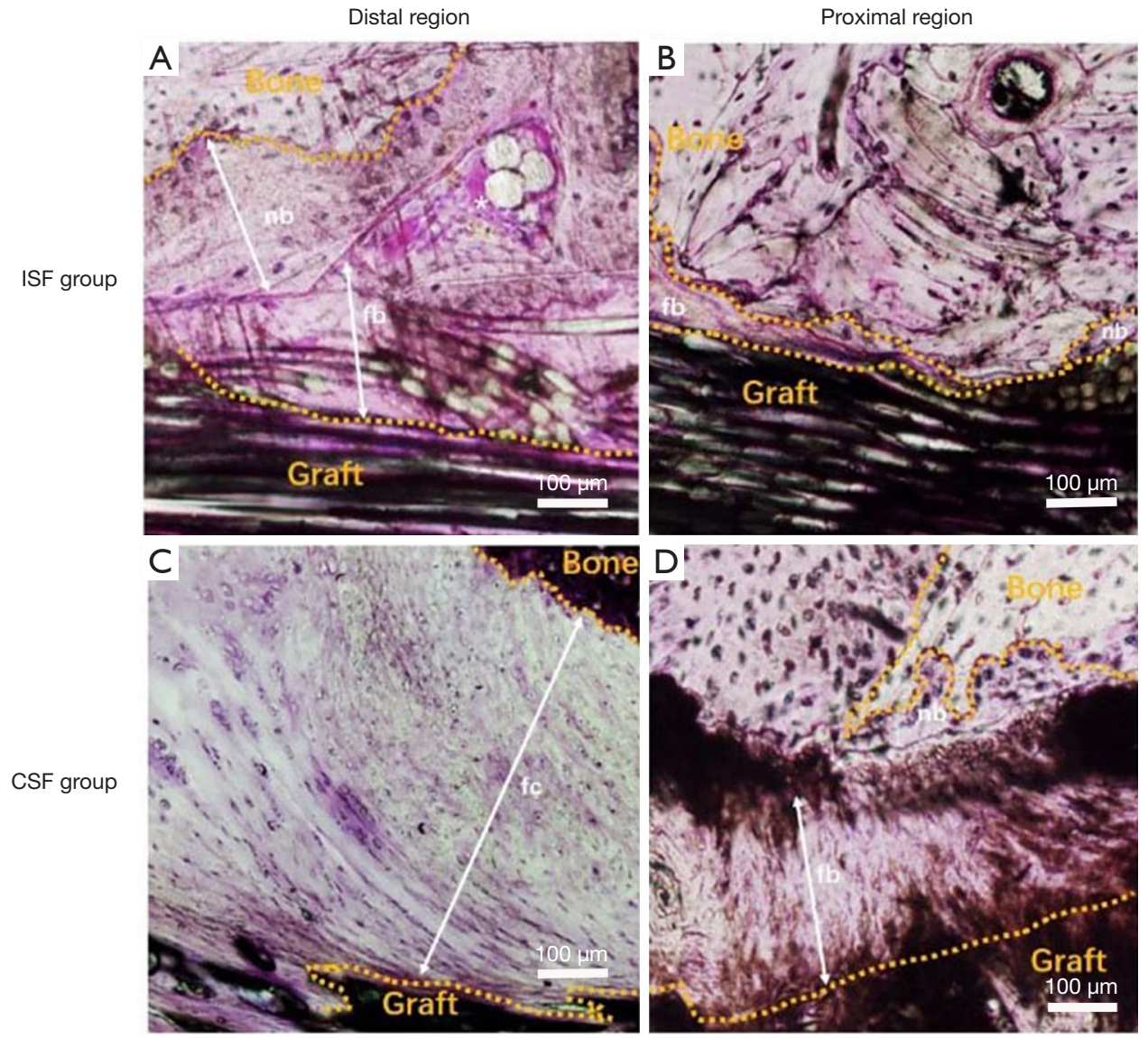

Figure 9 Femoral graft-bone interface at different location with Paragon staining at 3 months. (A) Distal region of the femoral tunnel of the ISF group; (B) proximal region of the femoral tunnel of the ISF group; (C) distal region of the femoral tunnel of the CSF group; (D) proximal region of the femoral tunnel of the CSF group. ISF, interference screw fixation; CSF, cortical suspensory fixation.

groups were $1,194 \pm 350$ and $1,097 \pm 764 \mathrm{~N}$, respectively, almost approaching the load-to-failure of the normal ACL $(1,254 \pm 350 \mathrm{~N})$. The graft-to-bone link was weak during the early postoperative time when using synthetic ligaments in ACLR (13). Thus, proper graft fixation is very important. The two fixation devices showed similar biomechanical results at 12 months. At 3 months, the CSF group appeared to have a higher load-to-failure than the ISF group, indicating that CSF may be a better choice to guarantee firm fixation for synthetic ligaments immediately following ACLR.

In this study, graft osseointegration was compared between ISF and CSF for ACL reconstruction in a sheep model at 3 or 12 months postoperatively. It was found that CSF promoted better graft-bone osseointegration of PET artificial ligament compared with ISF based on histological and CT results. The panorama of the graft-femoral tunnel complex in the ISF group displayed tunnel expansion in the tunnel aperture in comparison with the tunnel exit. It was previously believed that interference screw fixation could neutralize graft tunnel motion (32), while suspensory fixation could aggravate graft-tunnel motion and result in severe tunnel enlargement near the femoral tunnel aperture (33). However, our results argue against this theory.

In the present study, the ISF group revealed a larger femoral bone tunnel than the CSF group at 12 months after surgery according to the micro-CT scan. Previously, Boss et al. (34) found tunnel enlargement ranging from $30 \%$ to $100 \%$ at both 6 and 12 months after synthetic graft ACLR in a goat model. However, the pattern of bone tunnel enlargement was not described. Tunnel widening after ACLR is still a poorly understood phenomenon. Reported mechanical factors include tunnel malposition $(35,36)$, 

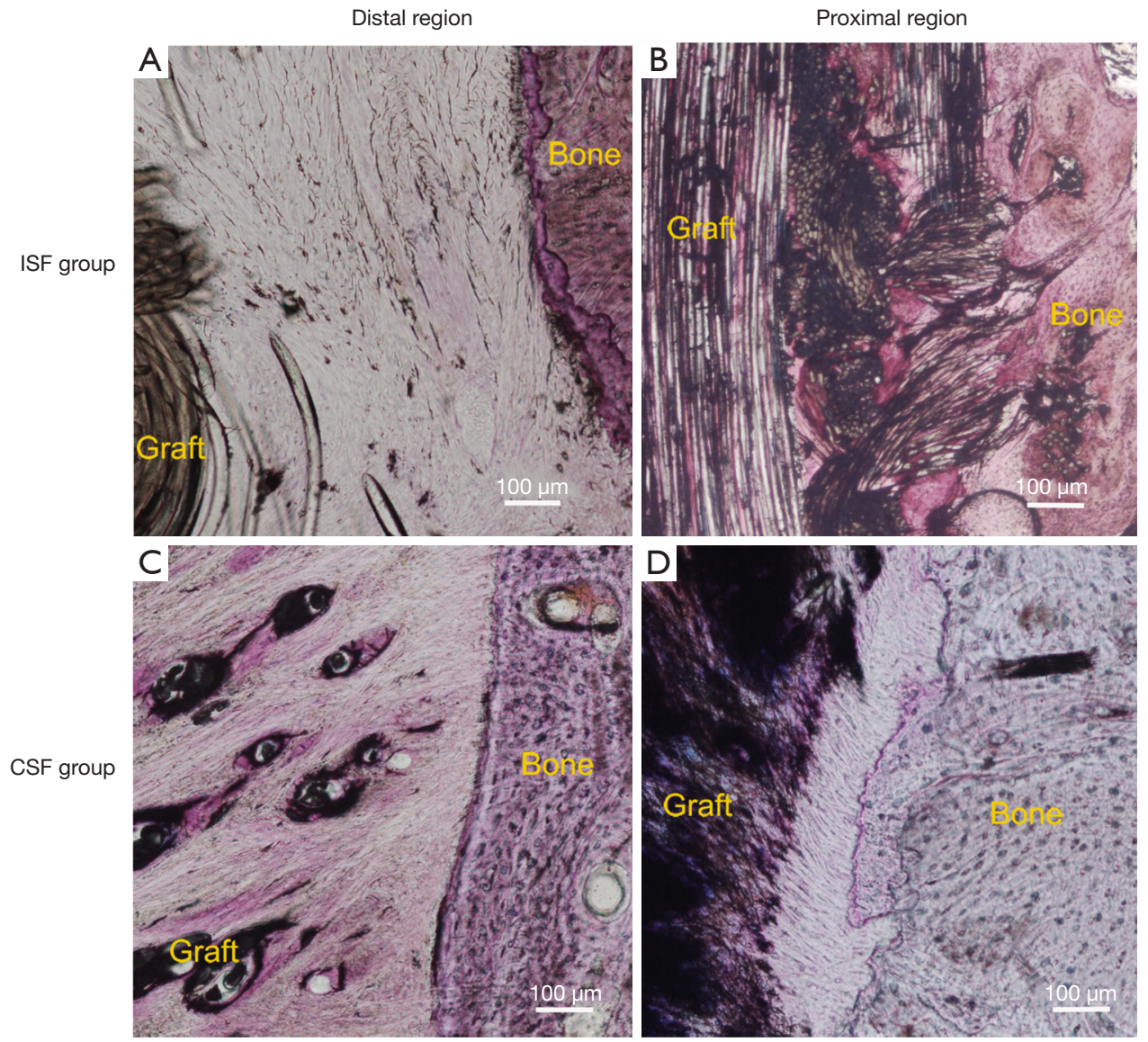

Figure 10 Femoral graft-bone interface at different location with Paragon staining at 12 months. (A) Distal region of the femoral tunnel of the ISF group; (B) proximal region of the femoral tunnel of the ISF group; (C) distal region of the femoral tunnel of the CSF group; (D) proximal region of the femoral tunnel of the CSF group. ISF, interference screw fixation; CSF, cortical suspensory fixation.

aggressive rehabilitation $(37,38)$, and improper fixation, all of which may correlate with increased graft-tunnel micromotion and graft tension (33). Biological factors include synovial fluid leakage, inflammatory response, remnant preservation, and osteoclast activation (39-41). Berg et al. (39) hypothesized that synovial fluid exposure, which might lead to "continuous exposure to enzymes, cytokines, and growth-factor inhibitors", could lead to enlargement of the tunnel aperture. During synthetic ligament reconstruction, the outside-in fixation method was used, and a space may easily form between the graft and the bone tunnel aperture, leaving it more susceptible to a "synovial bathing effect". A similar pattern of tunnel enlargement was found in clinical studies. Wang et al. (42) reported tunnel enlargement in the area without direct interference screw compression after autograft reconstruction. They ascribed the enlargement pattern to degenerated particles and activation of osteoclasts. Histological results from the ISF group showed giant cell infiltration in the region where the graft was not in direct contact with the screw. Therefore, we proposed that wear particles caused by inadequate fixation and subsequent foreign body immune response might precipitate tunnel aperture widening (38). In addition, tunnel aperture widening would exacerbate the transverse motion of the graft in the bone tunnel, known as the windshield-wiper effect (38), which might further aggravate bone tunnel enlargement.

The histological results of the present study demonstrate indirect graft-tunnel healing patterns, in accordance with previous studies of synthetic graft ACLR in large animal models. In a goat model of synthetic ligament ACLR, 
Boss et al. (34) found a thick fibrous interface around the prosthesis 6 months postoperatively, accompanied by new bone remodeling and collagenous fibers parallel to the longitudinal axis of the bone tunnel. Viateau et al. (13) found a fibrovascular layer at the graft-bone interface three months after synthetic ligament ACLR in sheep and described "Sharpey-like fibers" at the interface. They also described direct graft-to-bone contact in some sections. However, the region in which direct contact existed was not mentioned. Vaquette et al. (43) found fibrous encapsulation with intense cellularity and nonoriented fibers three months postoperatively in an ovine model. In some cases, direct graft-to-bone contact was also found. Cai et al. (40) investigated the effects of remnant preservation after reconstruction with PET ligament in beagle dogs and found that the graft-bone interface was filled with fibrous scar tissue three months postoperatively. According to a previous study (44), disoriented fibrous tissue exhibited inferior biomechanical strength and decreased load transfer due to its anisotropic composition, whereas fibers perpendicular to the bone surface were capable of load-bearing (25). In half of the histological specimens of the CSF group, the fibers were more regularly oriented and perpendicular to the bone interface at the tunnel aperture. Furthermore, fibrocartilage occurred near the femoral tunnel aperture when fixed with adjustable-loop cortical suspension devices in this study. This phenomenon has been reported in animal models of autograft ACLR (45). According to Weiler et al. (32), a fibrocartilaginous layer may form at the region where high intensity force exists, such as at the tunnel aperture. This might explain the findings from the CSF group.

This experiment has several limitations. Firstly, we did not remove the fixation systems for biomechanical testing. Therefore, it was unknown whether the difference in graft healing pattern had an effect on biomechanical strength. However, the results could prove that CSF and ISF provide equivalent fixation strength in the early stage of healing. Secondly, the sample size was relatively small for histological evaluation. Only four specimens were included in the histological evaluation. However, similarities in histological pattern were observed within the experimental groups. Finally, the biomechanical tests were made with knee extension rather than a degree of flexion.

\section{Conclusions}

No significant difference was found in biomechanical strength between the two fixation methods during the early stage of healing.

\section{Acknowledgments}

Funding: This project was subsidized by the Natural Science Foundation of China (81401812).

\section{Footnote}

Reporting Checklist: The authors have completed the ARRIVE reporting checklist. Available at https://dx.doi. org/10.21037/atm-21-1076

Data Sharing Statement: Available at https://dx.doi. org/10.21037/atm-21-1076

Conflicts of Interest: All authors have completed the ICMJE uniform disclosure form (available at https://dx.doi. org/10.21037/atm-21-1076). The authors declare that they have no competing interests.

Ethical Statement: The authors are accountable for all aspects of the work in ensuring that questions related to the accuracy or integrity of any part of the work are appropriately investigated and resolved. The animal experiments were performed under a project license (No. 201401050001) granted by Institutional Animal Care and Use Committee (IACUC) of Shanghai Jiao Tong University Animal Department, in compliance with national guidelines for the care and use of animals.

Open Access Statement: This is an Open Access article distributed in accordance with the Creative Commons Attribution-NonCommercial-NoDerivs 4.0 International License (CC BY-NC-ND 4.0), which permits the noncommercial replication and distribution of the article with the strict proviso that no changes or edits are made and the original work is properly cited (including links to both the formal publication through the relevant DOI and the license). See: https://creativecommons.org/ licenses/by-nc-nd/4.0/.

\section{References}

1. Tulloch SJ, Devitt BM, Porter T, et al. Primary ACL reconstruction using the LARS device is associated with a high failure rate at minimum of 6-year follow-up. Knee 
Surg Sports Traumatol Arthrosc 2019;27:3626-32.

2. Chen T, Zhang P, Chen J, et al. Long-Term Outcomes of Anterior Cruciate Ligament Reconstruction Using Either Synthetics With Remnant Preservation or Hamstring Autografts: A 10-Year Longitudinal Study. Am J Sports Med 2017;45:2739-50.

3. Kartus J, Movin T, Karlsson J. Donor-site morbidity and anterior knee problems after anterior cruciate ligament reconstruction using autografts. Arthroscopy 2001;17:971-80.

4. Stener S, Ejerhed L, Sernert N, et al. A longterm, prospective, randomized study comparing biodegradable and metal interference screws in anterior cruciate ligament reconstruction surgery: radiographic results and clinical outcome. Am J Sports Med 2010;38:1598-605.

5. Parchi PD, Gianluca C, Dolfi L, et al. Anterior cruciate ligament reconstruction with LARS ${ }^{\mathrm{TM}}$ artificial ligament results at a mean follow-up of eight years. Int Orthop 2013;37:1567-74.

6. Pan X, Wen H, Wang L, et al. Bone-patellar tendonbone autograft versus LARS artificial ligament for anterior cruciate ligament reconstruction. Eur J Orthop Surg Traumatol 2013;23:819-23.

7. Nau T, Lavoie P, Duval N. A new generation of artificial ligaments in reconstruction of the anterior cruciate ligament. Two-year follow-up of a randomised trial. J Bone Joint Surg Br 2002;84:356-60.

8. Liu ZT, Zhang XL, Jiang Y, et al. Four-strand hamstring tendon autograft versus LARS artificial ligament for anterior cruciate ligament reconstruction. Int Orthop 2010;34:45-9.

9. Tiefenboeck TM, Thurmaier E, Tiefenboeck MM, et al. Clinical and functional outcome after anterior cruciate ligament reconstruction using the $\mathrm{LARS}^{\mathrm{TM}}$ system at a minimum follow-up of 10 years. Knee 2015;22:565-8.

10. Ventura A, Terzaghi C, Legnani C, et al. Synthetic grafts for anterior cruciate ligament rupture: 19-year outcome study. Knee 2010;17:108-13.

11. Iliadis DP, Bourlos DN, Mastrokalos DS, et al. LARS Artificial Ligament Versus ABC Purely Polyester Ligament for Anterior Cruciate Ligament Reconstruction. Orthop J Sports Med 2016;4:2325967116653359.

12. Sinagra ZP, Kop A, Pabbruwe M, et al. Foreign Body Reaction Associated With Artificial LARS Ligaments: A Retrieval Study. Orthop J Sports Med 2018;6:2325967118811604.
13. Viateau V, Manassero M, Anagnostou F, et al. Biological and biomechanical evaluation of the ligament advanced reinforcement system (LARS AC) in a sheep model of anterior cruciate ligament replacement: a 3-month and 12-month study. Arthroscopy 2013;29:1079-88.

14. Guidoin MF, Marois Y, Bejui J, et al. Analysis of retrieved polymer fiber based replacements for the ACL. Biomaterials 2000;21:2461-74.

15. Yasuda K, Tsujino J, Tanabe Y, et al. Effects of initial graft tension on clinical outcome after anterior cruciate ligament reconstruction. Autogenous doubled hamstring tendons connected in series with polyester tapes. Am J Sports Med 1997;25:99-106.

16. Fleming B, Beynnon B, Howe J, et al. Effect of tension and placement of a prosthetic anterior cruciate ligament on the anteroposterior laxity of the knee. J Orthop Res 1992;10:177-86.

17. Zong JC, Ma R, Wang H, et al. The Effect of Graft Pretensioning on Bone Tunnel Diameter and Bone Formation After Anterior Cruciate Ligament Reconstruction in a Rat Model: Evaluation With Micro-Computed Tomography. Am J Sports Med 2017;45:1349-58.

18. Bianchi N, Sacchetti F, Bottai V, et al. LARS versus hamstring tendon autograft in anterior cruciate ligament reconstruction: a single-centre, single surgeon retrospective study with 8 years of follow-up. Eur J Orthop Surg Traumatol 2019;29:447-53.

19. Jia Z, Xue C, Wang W, et al. Clinical outcomes of anterior cruciate ligament reconstruction using LARS artificial graft with an at least 7-year follow-up. Medicine (Baltimore) 2017;96:e6568.

20. Chen J, Gu A, Jiang H, et al. A comparison of acute and chronic anterior cruciate ligament reconstruction using LARS artificial ligaments: a randomized prospective study with a 5-year follow-up. Arch Orthop Trauma Surg 2015;135:95-102.

21. Chan YS, Lo YP, Lien LC, et al. Improved divergence angles with femoral interference screw placement through the tibial tunnel as measured by multiplanar reconstruction computed tomography. Arthroscopy 2009;25:54-61.

22. Trentacosta N, Fillar AL, Liefeld CP, et al. Avoiding Complications and Technical Variability During Arthroscopically Assisted Transtibial ACL Reconstructions by Using a C-Arm With Image Intensifier. Orthop J Sports Med 
2014;2:2325967114530075.

23. Pierz K, Baltz M, Fulkerson J. The effect of Kurosaka screw divergence on the holding strength of bone-tendonbone grafts. Am J Sports Med 1995;23:332-5.

24. Chen W, Li H, Chen Y, et al. Bone-Patellar TendonBone Autografts Versus Hamstring Autografts Using the Same Suspensory Fixations in ACL Reconstruction: A Systematic Review and Meta-analysis. Orthop J Sports Med 2019;7:2325967119885314.

25. Smith PA, Stannard JP, Pfeiffer FM, et al. Suspensory Versus Interference Screw Fixation for Arthroscopic Anterior Cruciate Ligament Reconstruction in a Translational Large-Animal Model. Arthroscopy 2016;32:1086-97.

26. Colombet P, Graveleau N, Jambou S. Incorporation of Hamstring Grafts Within the Tibial Tunnel After Anterior Cruciate Ligament Reconstruction: Magnetic Resonance Imaging of Suspensory Fixation Versus Interference Screws. Am J Sports Med 2016;44:2838-45.

27. Mayr R, Smekal V, Koidl C, et al. ACL reconstruction with adjustable-length loop cortical button fixation results in less tibial tunnel widening compared with interference screw fixation. Knee Surg Sports Traumatol Arthrosc 2020;28:1036-44.

28. Goradia VK, Rochat MC, Kida M, et al. Natural history of a hamstring tendon autograft used for anterior cruciate ligament reconstruction in a sheep model. Am J Sports Med 2000;28:40-6.

29. Neddermann A, Willbold E, Witte F, et al. Tunnel widening after anterior cruciate ligament reconstruction: an experimental study in sheep. Am J Sports Med 2009;37:1609-17.

30. Teuschl AH, Tangl S, Heimel P, et al. Osteointegration of a Novel Silk Fiber-Based ACL Scaffold by Formation of a Ligament-Bone Interface. Am J Sports Med 2019;47:620-7.

31. Li H, Liu S, Sun Y, et al. Influence of Graft Bending Angle on Graft Maturation, the Femoral Tunnel, and Functional Outcomes by 12 Months After Anterior Cruciate Ligament Reconstruction. Orthop J Sports Med 2019;7:2325967119882663.

32. Weiler A, Hoffmann RF, Bail HJ, et al. Tendon healing in a bone tunnel. Part II: Histologic analysis after biodegradable interference fit fixation in a model of anterior cruciate ligament reconstruction in sheep. Arthroscopy 2002;18:124-35.

33. Rodeo SA, Kawamura S, Kim HJ, et al. Tendon healing in a bone tunnel differs at the tunnel entrance versus the tunnel exit: an effect of graft-tunnel motion? Am J Sports Med 2006;34:1790-800.

34. Boss JH, Shajrawi I, Soudry M, et al. Studies on a novel anterior cruciate ligament polyethylene fiber prosthesis: the histomorphological pattern of organization and bony anchorage of a polyethylene fiber prosthesis in the stifle of the goat. Clin Mater 1994;15:61-7.

35. Xu Y, Ao Y, Wang J, et al. Relation of tunnel enlargement and tunnel placement after single-bundle anterior cruciate ligament reconstruction. Arthroscopy 2011;27:923-32.

36. Ekdahl M, Nozaki M, Ferretti M, et al. The effect of tunnel placement on bone-tendon healing in anterior cruciate ligament reconstruction in a goat model. Am J Sports Med 2009;37:1522-30.

37. Hantes ME, Mastrokalos DS, Yu J, et al. The effect of early motion on tibial tunnel widening after anterior cruciate ligament replacement using hamstring tendon grafts. Arthroscopy 2004;20:572-80.

38. Höher J, Möller HD, Fu FH. Bone tunnel enlargement after anterior cruciate ligament reconstruction: fact or fiction? Knee Surg Sports Traumatol Arthrosc 1998;6:231-40.

39. Berg EE, Pollard ME, Kang Q. Interarticular bone tunnel healing. Arthroscopy 2001;17:189-95.

40. Cai J, Wan F, Ai C, et al. The Effect of Remnant Preservation on Tibial Tunnel Enlargement in Anterior Cruciate Ligament Reconstruction with Polyethylene Terephthalate Artificial Ligament in a Large Animal Model. J Knee Surg 2019;32:1094-101.

41. Rodeo SA, Kawamura S, Ma CB, et al. The effect of osteoclastic activity on tendon-to-bone healing: an experimental study in rabbits. J Bone Joint Surg Am 2007;89:2250-9.

42. Wang JH, Lee ES, Lee BH. Paradoxical tunnel enlargement after ACL reconstruction with hamstring autografts when using $\beta$-TCP containing interference screws for tibial aperture fixation- prospectively comparative study. BMC Musculoskelet Disord 2017;18:398.

43. Vaquette C, Viateau V, Guérard S, et al. The effect of polystyrene sodium sulfonate grafting on polyethylene terephthalate artificial ligaments on in vitro mineralisation and in vivo bone tissue integration. Biomaterials 2013;34:7048-63.

44. Liu Y, Birman V, Chen C, et al. Mechanisms of Bimaterial 
Attachment at the Interface of Tendon to Bone. J Eng Mater Technol 2011;133:011006.

45. Sato Y, Akagi R, Akatsu Y, et al. The effect of femoral

Cite this article as: Li H, Jiang F, Ge Y, Wan F, Li H, Chen S. Differences in artificial ligament graft osseointegration of the anterior cruciate ligament in a sheep model: a comparison between interference screw and cortical suspensory fixation. Ann Transl Med 2021;9(17):1370. doi: 10.21037/atm-21-1076 bone tunnel configuration on tendon-bone healing in an anterior cruciate ligament reconstruction: An animal study. Bone Joint Res 2018;7:327-35. 